\title{
BMJ Open What are determinants of utilisation of pharmaceutical anticancer treatment during the last year of life in Norway? A retrospective registry study
}

Christoffer Bugge (D) , ${ }^{1,2}$ Stein Kaasa, ${ }^{3}$ Erik Magnus Sæther, ${ }^{2}$ Hans Olav Melberg, ${ }^{1}$ Ivar Sonbo Kristiansen ${ }^{1,2}$

To cite: Bugge C, Kaasa S, Sæther EM, et al. What are determinants of utilisation of pharmaceutical anticancer treatment during the last year of life in Norway? A retrospective registry study. BMJ Open 2021;11:e050564. doi:10.1136/ bmjopen-2021-050564

- Prepublication history and additional supplemental material for this paper are available online. To view these files, please visit the journal online (http://dx.doi.org/10.1136/ bmjopen-2021-050564).

Presented at Society of Medical Decision Making (SMDM) meeting Chicago (virtual), October 2020.

Received 23 February 2021 Accepted 13 September 2021

Check for updates

(c) Author(s) (or their employer(s)) 2021. Re-use permitted under CC BY-NC. No commercial re-use. See rights and permissions. Published by BMJ.

${ }^{1}$ Department of Health Management and Health Economics, University of Oslo, Oslo, Norway

${ }^{2}$ Oslo Economics AS, Oslo, Norway

${ }^{3}$ Department of Oncology, Oslo University Hospital, Oslo, Norway

Correspondence to

Christoffer Bugge;

cbu@osloeconomics.no

\section{ABSTRACT}

Objectives The objective of this study was to investigate the use of, and predictors for, pharmaceutical anticancer treatment (PACT) towards the end of a patient's life in a country with a public healthcare system.

Design Retrospective registry study.

Setting Secondary care in Norway.

Participants All Norwegian patients with cancer (International Classification of Diseases tenth revision (ICD-10) codes C00-99, D00-09, D37-48) in contact with a somatic hospital in Norway between 2009 and 2017 $(\mathrm{N}=420655)$. Analyses were performed on a subsample of decedents with follow-back time of more than 1 year (2013-2017, N=52 496).

Interventions N/A.

Primary and secondary outcome measures Proportion of patients receiving PACT during the last year and month of life. We calculated Cls with block bootstrapping, while predictors of PACT were estimated with logistic regression. Results $24.0 \%$ (95\% Cl $23.4 \%$ to $24.6 \%$ ) of the patients received PACT during the last year of life and 3.2\% (95\% $\mathrm{Cl} 3.0 \%$ to $3.5 \%$ ) during their final month. The proportion during the last month was highest for multiple myeloma $(12.7 \%)$ and breast cancer (6.5\%) and lowest for urinary tract $(1.1 \%)$ and prostate and kidney cancer (1.4\%).

Patients living in northern (OR $0.80,95 \% \mathrm{Cl} 0.68$ to 0.94$)$ and western (OR $0.85,95 \% \mathrm{Cl} 0.75$ to 0.96 ) Norway had lower odds of PACT during the last month, while patients with myeloma (OR 3.0, 95\% Cl 2.5 to 3.7) and breast (OR $1.4,95 \% \mathrm{Cl} 1.1$ to 1.6) had higher odds. Kidney cancer (OR $0.25,95 \% \mathrm{Cl} 0.2$. to 0.4$)$, urinary tract $(\mathrm{OR} 0.38,95 \% \mathrm{Cl}$ 0.3 to 0.5 ) and prostate cancer (OR $0.4,95 \% \mathrm{Cl} 0.3$ to 0.5 ) were associated with lower probability of receiving PACT within the last month.

Conclusions The proportion of patients receiving PACT in Norway is lower than in several other industrialised countries. Age, type of cancer and area of living are significant determinants of variation in PACT.

\section{INTRODUCTION}

A fundamental problem for clinicians who consider prescribing end-of-life pharmaceutical anticancer treatment (PACT) is the assessment of the remaining lifetime of an individual patient and the expected patient

\section{STRENGTHS AND LIMITATIONS OF THIS STUDY}

$\Rightarrow$ Nationwide registry data during a period of 5 years with a range of key covariates.

$\Rightarrow$ No selection bias as virtually all patients with cancer in Norway receive medical care in public hospitals.

$\Rightarrow$ The data capture both hospital-administered and patient-administered pharmaceutical treatment.

$\Rightarrow$ The findings do not allow conclusions about whether patients receive too much or too little treatment.

$\Rightarrow$ Lack of information on disease stage, the treating clinician and comorbidities (possible explanatory variables).

benefit from the treatment. Whether oncologists should prescribe PACT near the end of life raises controversial ethical issues concerning the potential prolongation of life, quality of life for patient and their families, and the use of scarce healthcare resources. When such treatment is given near the end of life-the terminal phase, it may offer little potential to prolong life and is sometimes called futile-'serving no useful purpose, completely ineffective. ${ }^{\prime 2}$ The definition of the terminal phase has varied over time. Initially, terminal phase was defined as the time in which palliative care should be applied instead of active therapies. More recently it has become clear that palliative care should start earlier when active therapies are still in use. ${ }^{3}$ In this study, we explore those receiving PACT who are not in the terminal phase according to the first definition.

PACT may be associated with extensive adverse effects, and may impact the patient's ability to engage in meaningful life and prepare for death. ${ }^{4}$ It has been suggested that too much PACT in the last month of life may shorten life and, if side effects are not diagnosed and treated, reduce quality of life..$^{5-7}$ Still, the treatment requires funds and may displace beneficial treatment for others. In 
Norway, health authorities only fund new treatments that are cost-effective according to national guidelines and prioritising processes because budgets are not large enough to cover all treatments even if they are effective. ${ }^{8}$

Previous studies indicate that up to $38 \%$ of patients with cancer receive chemotherapy or other life-sustaining treatments during their last month of life. ${ }^{9}$ However, endof-life treatment decisions may differ across countries or within jurisdictions. ${ }^{9}$ In some healthcare systems, oncologists have an incentive to prescribe PACT because their remuneration depends on it. ${ }^{10}$ Such systems typically also impose a cost on patients in terms of copayments. In contrast, Norway has a public healthcare system in which oncologists have no personal financial incentive to prescribe, but also little disincentive in terms of patient co-payments (Norway has copayment on certain types of treatments, but a low total annual maximum of approximately US $\$ 270$ in 2020). It is, therefore, unclear which system results in the highest end-of-life PACT rates. The evidence of factors influencing end-of-life care in European countries is also lacking. In a 2014 systematic review of end-of-life studies in cancer care, Langton $e t$ a $t^{\natural}$ found 15 studies that examined quality indicators (including use of chemotherapy) for end-of-life care, of which none were from Europe. More recently, however, such studies have been published for Denmark and France. ${ }^{11}{ }^{12}$ The proportion receiving chemotherapy during the last 14 days of life was $4.2 \%$ in Denmark and $11.3 \%$ in France. ${ }^{112}$

Use of PACT towards the end of life represents a difficult medical decision and an important policy issue for patients and society. One approach to improve the quality in end-of-life care is to gain a better understanding of current patterns of end-of-life treatment at a national level in a public healthcare system where the overall goal is to offer patients equitable access to care. We used comprehensive individual patient-level nationwide data from the Norwegian Patient Registry ${ }^{13}$ to answer two research questions: First, what proportion of patients receive PACT during their last year and months of life in Norway? Second, to what extent are treatment decisions influenced by patients' age, gender, type of cancer or geographical factors?

\section{METHODS}

We performed a retrospective cohort study using data from the Norwegian Patient Registry (delivered August 2018). Each episode of care (inpatient stay, day care or outpatient visit) in the Norwegian Patient Registry has a main International Classification of Diseases Tenth Revision (ICD-10) diagnostic code, possibly supplementary diagnostic codes, and a unique patient identifier that allows patients to be followed over time. Diagnostic codes in the Norwegian Patient Registry have proved valid when compared with the Cancer Registry of Norway. ${ }^{14}$ Each episode of care also had a diagnosis-related groups (DRG) code and an Anatomical Therapeutic Chemical (ATC) code in cases where PACT was administered. Additionally, the dataset encompassed the following variables: unique patient ID, patient age and gender, county of residence (19 counties), year/month of episode, medical and surgical procedure codes, code for infusion of PACT, days until death and level of care (hospital outpatient/day care and inpatient).

We included all episodes of care with ICD-10 codes C00-99, D00-09, D37-48. Norway has a population of approximately 5.4 million, and in 2017, a total of 33564 new cancer cases were reported and 273741 Norwegians were alive after having received a cancer diagnosis. ${ }^{15}$ Cancer treatment in hospice and private hospitals is negligible, thus, the Norwegian Patient Registry includes virtually all Norwegian patients with cancer. Our dataset encompassed 7423828 episodes for 420655 patients. Our data included all patients with cancer who had been in contact with Norwegian hospitals (outpatient or inpatient) between 2009 and 2017 among whom 128413 were reported dead by the end of 2017. All analyses were performed on a subset of the data (a 5 year cohort of patients who died during 2013-2017 with at least 1-year follow-back data, $\mathrm{N}=52496$ ) as patient administered treatment (oral and subcutaneous) were not identifiable in the registry before 2013 (see figure 1). Patient characteristics for the complete sample for the period 2013-2017 and the subsample used for analyses are presented in online supplemental table S1. The Norwegian Patient Registry has systems for data cleaning and continually check for correctness. We have further checked for correctness and consistency without detecting need for further cleaning.

\section{Classification of cancer diagnosis}

Patients with more than one cancer diagnosis were classified into mutually exclusive cancer diagnoses based on their main and supplementary diagnosis (online supplemental table S1). Patients with multiple cancer diagnoses were assigned to a single diagnosis based on their most frequently listed cancer diagnosis type. Classification of cancer diagnosis was based on data from 2009 to 2017.

\section{Pharmaceutical anticancer treatment}

PACT included cytostatic agents, cytotoxic agents, targeted therapies and immunotherapies. We classified PACT as intravenous, subcutaneous or oral by means of ATC codes and the DRG system. To calculate the proportion of patient who received PACT 1 year before death, patients with follow-back time less than 1 year from first episode of cancer until death were excluded from the analyses. We ran additional analyses to investigate the effect of limiting our study population to patients with at least 1-year follow-back.

\section{Statistical analysis}

For each patient, we registered whether he or she received PACT during the following periods prior to death: 12, 9, 6 and 3 months, and 1, 2, 3, 4, 5, 6, 7 and 8 weeks. Proportion of patients receiving PACT were defined as $\frac{P A C T_{t}}{N}$ 


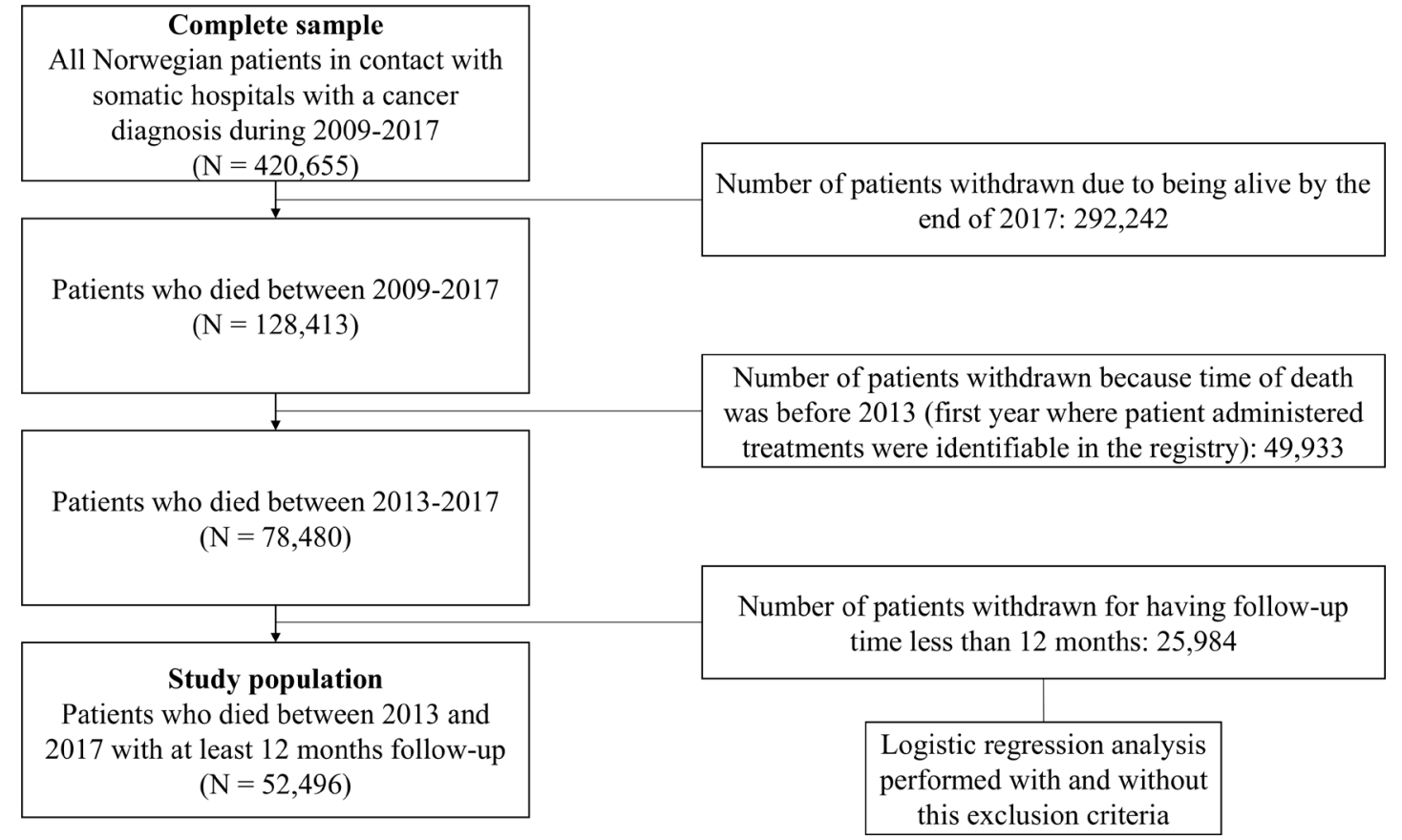

Figure 1 Flow diagram for selection of study population.

where $P A C T_{t}$ is the number of patients who received PACT during time period $t$ ( $t=$ time period prior to death and $N$ is the number of patients with more than 12 months follow-back time before death. In other words, the numbers present aggregates over time periods, not treatment at a point in time.

We used block bootstrapping to estimate the SE of the proportion of patients who received PACT during time period $t{ }^{16}$ To reproduce the dependence structure of the observed data in the resampled data, we created blocks of consecutive data defined as each individual patient's treatment course (last year before death). We estimated logistic regression models to identify predictors of receiving PACT during the last month of life. The following variables were included in the multivariable regression model: year of death, patient's age at death, region of hospital, gender and type of cancer. The variables were chosen because they were expected to influence the use of PACT and due to availability in the obtained data.

All analyses were performed using STATA software V.14 (StataCorp).

\section{Patient and public involvement}

No patient involved.

\section{RESULTS}

\section{Use of end-of-life PACT}

A total of 301611 patients with cancer received care in a hospital during the period 2013-2017. Of these patients, 52496 patients $(17.4 \%)$ were reported dead by the end of 2017 and had at least 1-year follow-back. Of this group, $12604(24.0 \%, 95 \%$ CI $23.4 \%$ to $24.6 \%)$ received PACT during the last year of life (figure 2). The rates of PACT 1 year prior to death were highest for pancreatic cancer $(60.7 \%, 95 \%$ CI $58.0 \%$ to $63.5 \%)$, multiple myeloma $(53.0 \%, 95 \%$ CI $50.2 \%$ to 55.8$)$ and lung cancer $(45.7 \%$, $95 \%$ CI $44.4 \%$ to $47.1 \%)$. Kidney cancer $(11.7 \%$, $95 \%$ CI $9.6 \%$ to $13.7 \%)$, urinary tract $(12.8 \%, 95 \%$ CI $11.6 \%$ to $14.0 \%)$ and leukaemia $(14.4 \%, 95 \%$ CI $3.1 \%$ to $15.8 \%)$ had low PACT rates during last year of life. In total 1691 (3.2\%, 95\% CI $3.0 \%$ to $3.5 \%)$ received PACT at least once during the last month before death, and patients with multiple myeloma $(12.7 \%, 95 \%$ CI $10.9 \%$ to $14.5 \%)$, breast $(6.5 \%, 95 \%$ CI $5.7 \%$ to $7.3 \%)$ and mouth/pharynx cancer $(6.0 \%, 95 \%$ CI $4.3 \%$ to $7.7 \%)$ had the highest rates. Among cancers with low PACT rates during the last month before death were urinary tract $(1.1 \%, 95 \%$ CI $0.7 \%$ to $1.5 \%)$, kidney cancer $(1.4 \%, 95 \%$ CI $0.7 \%$ to $2.0 \%)$ and prostate cancer $(1.4 \%, 95 \%$ CI $1.1 \%$ to $1.6 \%)$.

\section{Predictors of variation in treatment decisions}

The odds for receiving PACT during the last month of life were highest for patients aged 40-59 years and lowest for those aged $80+($ table 1$)$. Adjusted for the included covariates, patients living in Northern (OR 0.80, 95\% CI 0.68 to 0.94 ) or Western Norway (OR $0.85,95 \%$ CI 0.75 to 0.96 ) had lower odds of receiving PACT within the last month of life compared with those living in the South-East region. Also, patients diagnosed with multiple myeloma (OR 3.03, 95\% CI 2.48 to 3.72) and breast cancer (OR 1.36, 95\% CI 1.13 to 1.63) had higher odds of receiving PACT during the last month of life (compared with lung cancer). Kidney cancer (OR $0.25,95 \%$ CI 0.15 to 0.43 ), urinary tract (OR $0.38,95 \%$ CI 0.27 to 0.53 ) and prostate cancer (OR $0.39,95 \%$ CI 0.31 to 0.49 ) had significantly lower odds. There was no clear trend towards 


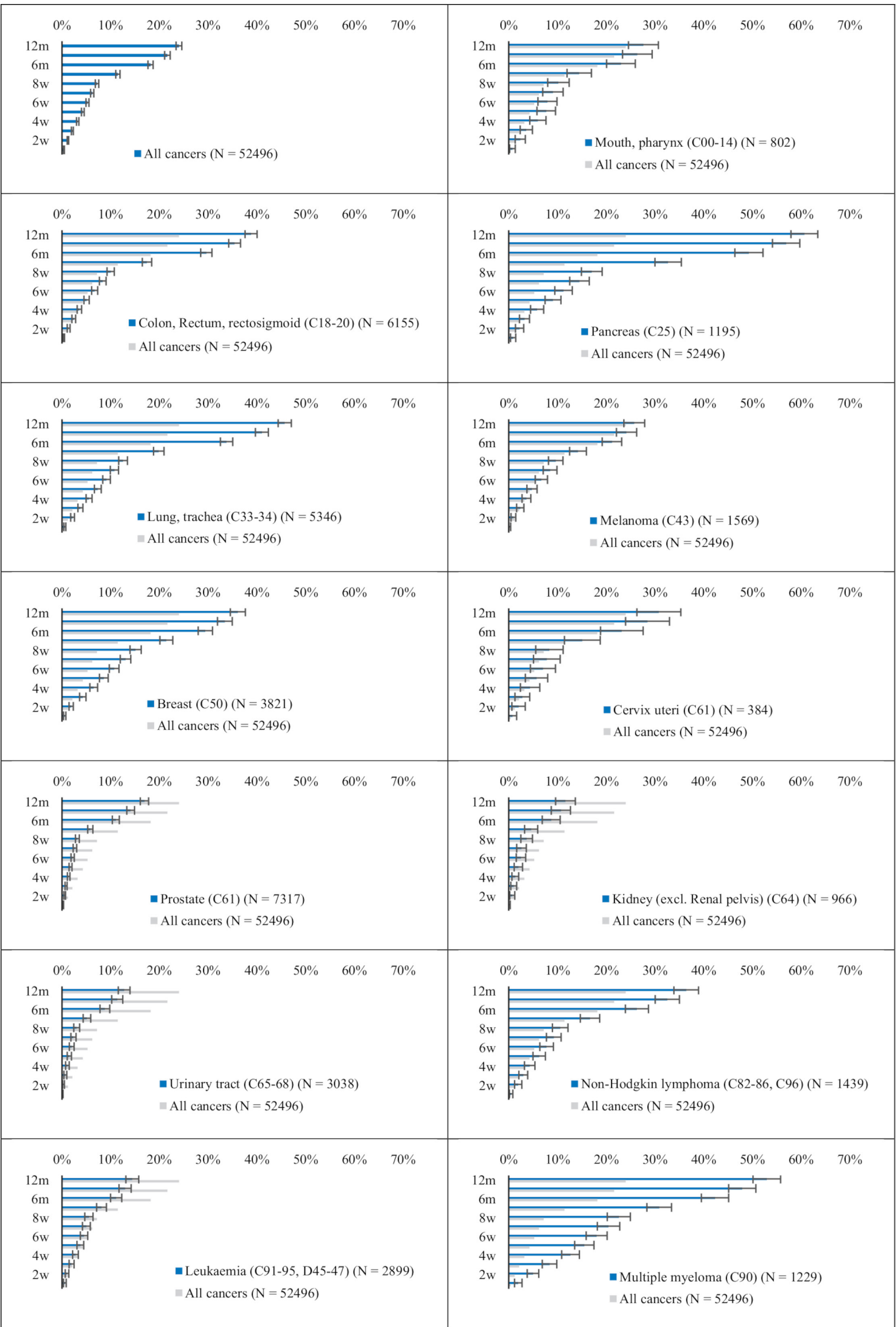

Figure 2 Proportion of patients with cancer receiving pharmaceutical anticancer treatment during last weeks or months of life, 2013-2017. 
Table 1 Multivariable logistic regression analysis of the odds that patients received pharmaceutical anticancer treatment last month before death

\begin{tabular}{|c|c|c|}
\hline & OR (95\% Cl) & $P$ value \\
\hline \multicolumn{3}{|l|}{ Year of death } \\
\hline Reference: 2013 & 0.99 (0.96 to 1.03$)$ & 0.74 \\
\hline \multicolumn{3}{|l|}{ Age } \\
\hline 60-69 years (reference) & 1.00 & NA \\
\hline $0-9$ years & 0.85 (0.20 to 3.52$)$ & 0.82 \\
\hline $10-19$ years vs $60-69$ years & 0.74 (0.23 to 2.38$)$ & 0.62 \\
\hline $20-29$ years vs $60-69$ years & $0.68(0.29$ to 1.55$)$ & 0.36 \\
\hline $30-39$ years vs $60-69$ years & 1.08 (0.73 to 1.61$)$ & 0.70 \\
\hline $40-49$ years vs $60-69$ years & 1.46 (1.19 to 1.78$)$ & $<0.001$ \\
\hline $50-59$ years vs $60-69$ years & 1.33 (1.16 to 1.53$)$ & $<0.001$ \\
\hline $70-79$ years vs $60-69$ years & $0.62(0.55$ to 0.69$)$ & $<0.001$ \\
\hline $80-89$ years vs $60-69$ years & 0.14 (0.12 to 0.17$)$ & $<0.001$ \\
\hline $90-99$ years vs $60-69$ years & $0.03(0.14$ to 0.53$)$ & $<0.001$ \\
\hline \multicolumn{3}{|c|}{ Hospital affiliation (regional health authority) } \\
\hline South-Eastern (reference) & 1.00 & NA \\
\hline Central & $1.02(0.90$ to 1.17$)$ & 0.74 \\
\hline Western & 0.85 (0.75 to 0.96$)$ & 0.01 \\
\hline Northern & 0.80 (0.68 to 0.94$)$ & 0.01 \\
\hline \multicolumn{3}{|l|}{ Gender } \\
\hline Women & 0.97 (0.87 to 1.07$)$ & 0.52 \\
\hline \multicolumn{3}{|l|}{ Type of cancer } \\
\hline Lung cancer (reference) & 1.00 & NA \\
\hline Mouth, pharynx & 1.14 (0.84 to 1.55$)$ & 0.41 \\
\hline Colon, rectum, rectosigmoid & 0.84 (0.71 to 0.99$)$ & 0.05 \\
\hline Pancreatic & 1.21 (0.94 to 1.54$)$ & 0.14 \\
\hline Melanoma & 0.77 (0.58 to 1.03$)$ & 0.08 \\
\hline Breast & 1.36 (1.13 to 1.63$)$ & $<0.001$ \\
\hline Cervix uteri & 0.69 (0.42 to 1.13$)$ & 0.14 \\
\hline Prostate & 0.39 (0.31 to 0.49$)$ & $<0.001$ \\
\hline Kidney (excl. renal pelvis) & 0.25 (0.15 to 0.43$)$ & $<0.001$ \\
\hline Urinary tract & 0.38 (0.27 to 0.53$)$ & $<0.001$ \\
\hline Non-Hodgkin's lymphoma & 1.06 (0.81 to 1.38$)$ & 0.69 \\
\hline Leukaemia & 0.72 (0.57 to 0.93$)$ & 0.01 \\
\hline Multiple myeloma & 3.03 (2.48 to 3.72 ) & $<0.001$ \\
\hline Residual group* & 0.66 (0.57 to 0.76$)$ & $<0.001$ \\
\hline
\end{tabular}

Constant: $0.10(0.08-0.11)$.

${ }^{*}$ Residual group includes all cancers not presented above. NA, not applicable; OR, odds ratio.

higher or lower provision of end-of-life PACT during the observation period and we found no differences between genders.

Additionally, we ran analyses for women and men separately and analyses excluding cancers that only occur for one gender (breast, uterine, prostate), but we were not able to find any differences between genders. Also, we tested for several plausible interactions between gender, age, health region and cancer type. In total, we tested for
36 interactions. Six were borderline significant, but none of the interactions were meaningful (online supplemental table S2). Finally, we investigated the impact of restricting our population to patients with at least a 1-month follow back period (compared with 1 year in our base-case analysis). The predictors were not substantially affected by the change in patient population (online supplemental table S3).

\section{DISCUSSION}

One in four Norwegian patients with cancer receive PACT during their last year of life, while $3 \%$ are treated within the last month. Patients who are elderly or residents of Northern or Western Norway (regions with a lower population density compared with the south-east region) are less likely to receive PACT during the last month of life. The proportion receiving PACT end-of-life in Norway are relatively low compared with other European countries with similar healthcare systems, indicating that medical culture and patient preference impact choice of treatment end of life.

Previous studies indicate high levels of PACT use near the end of life with rates up to $38 \%$ during last month in countries in North America, Asia and Europe. ${ }^{91117-24}$ Our findings of generally low PACT rates in Norway align well with results reported previously ${ }^{23}$ and the relative low rates found in our neighbouring country Denmark ${ }^{12}$ which has a similar healthcare system. Having a public healthcare system, where oncologist have no financial incentive to prescribe, may explain the lower rates in Norway. Additionally, due to strict priority setting for pharmaceuticals, not all new treatment options are available to Norwegian patients. Other factors may also explain the lower rates, such as the role of palliative care teams or culture and attitude towards end-of-life treatment in the clinical environments. Even if the negligible patient copayments in Norway should favour use of PACT, the inhibitive factors dominate the final decisions. Our findings support previous research that factors such as age, tumour site and region of residence influence the level of PACT near death. 112024

The main strength of our study lies in the use of nationwide registry data for a period of 5 years with a range of key covariates. Almost all patients with cancer in Norway receive medical care in public hospitals, so our data cover virtually the entire Norwegian population (no selection bias). Our data also include a range of covariates allowing adjustment for patient characteristics, year of treatment, type of cancer and patient's place of living. Another strength is that we capture both hospital-administered and patient-administered PACT. In recent years, subcutaneous and oral PACT have become important modalities, making it essential to include these treatments when studying end-of-life care.

However, the study also has several limitations. First, we do not have information on disease stage, the treating clinician, comorbidities or functional status. Complete 
information of ATC codes was also not available; thus, classes of PACT were not considered as determinant in the analyses. A key challenge of the study design is that we cannot translate the findings directly into how to change clinical practice. Second, there is some risk of misclassification of diagnosis. We chose patients' most frequent diagnosis but tested alternative algorithms for assigning diagnosis. In $90 \%$ of the cases the same cancer diagnosis was assigned regardless of which algorithm had been applied. For legal reasons, our diagnosis data could not be confirmed by linkage to the cancer registry, but diagnostic codes in the Norwegian Patient Registry have proved valid when compared with the Cancer Registry of Norway. ${ }^{14}$ Even though some patients may have been assigned an incorrect diagnosis, it is unlikely that this would substantially impact the analysis of predictors. Third, we do not have information on the cause of death and assumed that all patients died from their cancer. We analyse a subsample of descendants between 2013 and 2017 (52 496 patients), which correspond well to the number of cancer deaths in Norway during this period in the cause of death registry (54 204 deaths). Lastly, retrospective studies like this one may create a biased portrait of terminal care because of the way subjects are identified and the time periods that are examined. ${ }^{25}$ To address this challenge, we ran additional analyses illustrating that our results only were marginally affected by changes in the study population. Our analyses are not based on any specific definition of terminal phase, because we only have information on the time of death, not the intention of the treatment given.

Clinicians' choice of end-of-life treatment may be influenced by characteristics of the provider (supply factors) and of the patients, their dependents and society in general (demand factors). On the supply side, clinicians may be affected by their department's culture, their training, experience and marketing from the pharmaceutical industry. Additionally, payment systems and reimbursement schemes have been found to influence clinicians' use of PACT ${ }^{26}$ The regional differences found in our study may indicate that department culture and training may influence end-of-life treatment. Also, distance to hospital may play a role as travel distances are less in the south-east region of Norway than others. However, it is not necessarily the case that lower use of PACT during the patient's final months imply better or more appropriate care.

Better knowledge on end-of-life treatment and factors influencing the use of PACT are important in order to ensure optimal treatment to avoid undue suffering among patients and their dependents and unnecessary use of healthcare resources.

\section{CONCLUSION}

Use of chemotherapy near end of life is modest in the Norwegian healthcare system with universal access to care and minimal patient copayment. Several other countries with similar systems have higher PACT rates during the last months of life, which indicate that not only financial incentives, but also medical culture and patient preferences may impact choice of treatment. Information on PACT rates may be useful for clinicians in order to achieve optimal end-of-life care.

Contributors CB: Data collection, study design, analysis, interpretation of results, preparation of the manuscript and approval of the manuscript. SK: Study design, interpretation of results, preparation of the manuscript and approval of the manuscript. EMS: Data collection, interpretation of results, preparation of the manuscript and approval of the manuscript. HOM: Interpretation of results, preparation of the manuscript and approval of the manuscript. ISK: Data collection, study design, interpretation of results, preparation of the manuscript and approval of the manuscript.

Funding This work was supported by the Norwegian Research Council (grant number: 272493). Data from the Norwegian Patient Registry were originally funded by Bristol Myers Squibb (Norway) for another project conducted by Oslo Economics AS.

Disclaimer The funders had no role in the design and conduct of the study; in the data collection, analysis, or; interpretation of results in the preparation, review, or approval of the manuscript.

Competing interests CB, EMS and ISK are affiliated with Oslo Economics and have all completed consultancy assignments for several pharmaceutical companies in recent years. None of the projects have been related to the work presented in this paper.

Patient consent for publication Not applicable.

Ethics approval The Norwegian Data Inspectorate (17/00565-2/CDG) and the Regional Committees for Medical and Health Research Ethics (2017/769/REK) granted approval for the use of data from the Norwegian Patient Registry.

Provenance and peer review Not commissioned; externally peer reviewed.

Data availability statement Data are available upon reasonable request. Data may be obtained from a third party and are not publicly available. The study uses registry data from the Norwegian Patient Registry. Individual patient level data can be obtained by application to the registry. Authors are willing to share access to the STATA programming code (do-file). Individual patient level data are protected by law, and reviewers have to come to Oslo to have access to data.

Supplemental material This content has been supplied by the author(s). It has not been vetted by BMJ Publishing Group Limited (BMJ) and may not have been peer-reviewed. Any opinions or recommendations discussed are solely those of the author(s) and are not endorsed by BMJ. BMJ disclaims all liability and responsibility arising from any reliance placed on the content. Where the content includes any translated material, BMJ does not warrant the accuracy and reliability of the translations (including but not limited to local regulations, clinical guidelines, terminology, drug names and drug dosages), and is not responsible for any error and/or omissions arising from translation and adaptation or otherwise.

Open access This is an open access article distributed in accordance with the Creative Commons Attribution Non Commercial (CC BY-NC 4.0) license, which permits others to distribute, remix, adapt, build upon this work non-commercially, and license their derivative works on different terms, provided the original work is properly cited, appropriate credit is given, any changes made indicated, and the use is non-commercial. See: http://creativecommons.org/licenses/by-nc/4.0/.

ORCID iD

Christoffer Bugge http://orcid.org/0000-0002-0766-2870

\section{REFERENCES}

1 Cayne BS. The new lexicon Webster's dictionary of the English language: Outlet 1987.

2 Kasman DL. When is medical treatment futile? A guide for students, residents, and physicians. J Gen Intern Med 2004;19:1053-6.

3 Hausner D, Tricou C, Mathews J, et al. Timing of palliative care referral before and after evidence from trials supporting early palliative care. Oncologist 2021;26:332-40.

4 Wagner AD, Grothey A, Andre T, et al. Association of sex and adverse events (AEs) of adjuvant chemotherapy (ACT) in early stage 
colon cancer (CC): a pooled analysis of 28,636 patients (pts) in the ACCENT database. J Clin Oncol 2018;36:3603-03.

5 Temel JS, Greer JA, Muzikansky A, et al. Early palliative care for patients with metastatic non-small-cell lung cancer. N Engl J Med 2010;363:733-42.

6 Basch E, Deal AM, Kris MG, et al. Symptom monitoring with patientreported outcomes during routine cancer treatment: a randomized controlled trial. J Clin Oncol 2016;34:557-65.

7 Basch E, Schrag D. The Evolving Uses of "Real-World" Data. JAMA 2019;321:1359-60.

8 Ministry of Health and Care Services. White paper (Meld. St. 34 (2015-2016) on priority setting in the health service [in Norwegian]. Oslo Ministry of Health and Care Services; 2016.

9 Langton JM, Blanch B, Drew AK, et al. Retrospective studies of end-of-life resource utilization and costs in cancer care using health administrative data: a systematic review. Palliat Med 2014;28:1167-96.

10 Mitchell AP, Rotter JS, Patel E, et al. Association between reimbursement incentives and physician practice in oncology: a systematic review. JAMA Oncol 2019;5:893-9.

11 Rochigneux P, Raoul JL, Beaussant Y, et al. Use of chemotherapy near the end of life: what factors matter? Ann Oncol 2017;28:809-17.

12 Skov Benthien K, Adsersen M, Petersen MA, et al. Is specialized palliative cancer care associated with use of antineoplastic treatment at the end of life? a population-based cohort study. Palliat Med 2018;32:1509-17.

13 Bakken IJ, Surén P, Håberg SE, et al. Norsk pasientregister - en viktig kilde for forskning. Tidsskriftet 2014;134:12-13.

14 Bakken IJ, Gystad SO, Christensen Øyvind Olav Schjøtt, et al. Comparison of data from the Norwegian patient register and the cancer registry of Norway. Tidsskr Nor Laegeforen 2012;132:1336-40.

15 Cancer Registry of Norway. Cancer in Norway 2017 - Cancer incidence, mortality, survival and prevalence in Norway. Oslo Cancer Registry of Norway; 2018.
16 Lahiri SN. Theoretical comparisons of block bootstrap methods. The Annals of Statistics 1999;27:386-404.

17 Michael N, Beale G, O'Callaghan C, et al. Timing of palliative care referral and aggressive cancer care toward the end-of-life in pancreatic cancer: a retrospective, single-center observational study. BMC Palliat Care 2019;18:13.

18 Emanuel EJ, Young-Xu Y, Levinsky NG, et al. Chemotherapy use among Medicare beneficiaries at the end of life. Ann Intern Med 2003;138:639-43.

19 Earle CC, Neville BA, Landrum MB, et al. Trends in the aggressiveness of cancer care near the end of life. $J$ Clin Oncol 2004;22:315-21.

20 Earle CC, Landrum MB, Souza JM, et al. Aggressiveness of cancer care near the end of life: is it a quality-of-care issue? $\mathrm{J}$ Clin Oncol 2008;26:3860-6.

21 Braga S. Why do our patients get chemotherapy until the end of life? Ann Oncol 2011;22:2345-8.

22 Yun YH, Kwak M, Park SM, et al. Chemotherapy use and associated factors among cancer patients near the end of life. Oncology 2007;72:164-71.

23 Bekelman JE, Halpern SD, Blankart CR, et al. Comparison of site of death, health care utilization, and hospital expenditures for patients dying with cancer in 7 developed countries. JAMA 2016;315:272-83.

24 Matter-Walstra KW, Achermann R, Rapold R, et al. Delivery of health care at the end of life in cancer patients of four Swiss cantons: a retrospective database study (SAKK 89/09). BMC Cancer 2014;14:306.

25 Bach PB, Schrag D, Begg CB. Resurrecting treatment histories of dead patients: a study design that should be laid to rest. JAMA 2004;292:2765-70.

26 Colla CH, Morden NE, Skinner JS, et al. Impact of payment reform on chemotherapy at the end of life. J Oncol Pract 2012;8:e6s-13. 